\title{
Introducing a Novel Method for Three Dimensional Dose Map by Optical Computed Tomography
}

\author{
Nooshin Banaee, ${ }^{1}$ Hassan Ali Nedaie,,${ }^{2,3,}$ Alireza Shirazi, ${ }^{3}$ Alireza Zirak, ${ }^{4}$ and Sodeh Sadjadi ${ }^{5}$ \\ ${ }^{1}$ Department of Engineering, Science and Research Branch, Islamic Azad University, Tehran, Iran \\ ${ }^{2}$ Joint Cancer Research Center, Radiotherapy Oncology \& Radiobiology Research Center, Cancer Institute, Tehran University of Medical Sciences, Tehran, Iran \\ ${ }^{3}$ Department of Medical Physics and Biomedical Engineering, Faculty of Medicine and Radiation Oncology Research Center Cancer Institute, Tehran University of Medical \\ Sciences, Tehran, Iran \\ ${ }^{4}$ Laser and Optics Research School, Nuclear Science and Technology Research Institute, Tehran, Iran \\ ${ }^{5}$ Nuclear Science and Technology Research Institute, Tehran, Iran \\ "Corresponding author: Hassan Ali Nedaie, Joint Cancer Research Center, Radiotherapy Oncology \& Radiobiology Research Center, Cancer Institute, Tehran University of \\ Medical Sciences, Tehran, Iran. Tel/Fax: +98-21 66948673, E-mail: nedaieha@sina.tums.ac.ir
}

Received 2016 February 22; Revised 2016 April 22; Accepted 2016 May 09.

\begin{abstract}
Background: Radiochromic gel is recently introduced as a three dimensional dosimeter that exhibits color changes by being exposed to ionizing radiation. Therefore, the read-out system of this dosimeter should be based on radiochromic response.

Objectives: The aim of this study was to introduce a novel design of machine called optical computed tomography (OCT) and acquire the three dimensional dose distribution of ionizing radiation by using the Radon transform image reconstruction technique.

Materials and Methods: By assembling red light-emitting diode as a light source, two lenses, charge-coupled device (CCD) camera, step motor, matching liquid, radiochromic gel, and a base frame, a novel design of OCT was manufactured. Then a sample of radiochromic gel was irradiated and scanned by novel OCT. The acquired images were then reconstructed by using the Radon transform algorithm and consequently the dose distribution was mapped. Finally the results of this device were verified by an ion chamber.

Results: By setting up the required accessories, a new design of OCT was constructed. The verification test showed that the results of novel OCT could be reliable by almost $\pm 3 \%$ deviations.

Conclusion: The results of this study confirmed that not only the Radon transform algorithm could be used for reconstructing computed tomography (CT) images, but also this technique could be applied for imaging the dose map of ionizing radiation obtained by OCT. Moreover, novel OCTradiochromic gel could be considered as a proper system for acquiring three dimensional dose distribution of ionizing radiation.
\end{abstract}

Keywords: Optical Computed Tomography, Dosimetry, Radon Transform, Dose Distribution

\section{Background}

International atomic energy agency (IAEA) safety standards recommends being equipped with a qualified dosimeter in terms of water equivalency, no-energy dependence, low fading, high sensitivity, providing three dimensional dose information and also high spatial resolution when working with ionizing radiations (1).

Recently, a novel transparent polymer, known as radiochromic gel has been introduced for three dimensional dose measurements. This gel is a polyurethane material doped with leuco dyes that exhibits a radiochromic response when exposed to ionizing radiation. The relationship between dose and optical changes of the gel is demonstrated as linear (2). Therefore, this dosimeter needs a readout system based on color changes.

\section{Objectives}

The aim of this study was to introduce a novel design of the optical computed tomography (OCT) machine for imaging the dose distribution of ionizing radiation exposed to water equivalent radiochromic gel dosimeter by using the Radon transform image reconstruction technique.

\section{Materials and Methods}

\subsection{Radiochromic Gel Fabrication}

At onset of the study, the water equivalent radiochromic gel dosimeter was fabricated. The procedure consisted of mixing CCl4 (Merck, Keinlworth, United States) and leuco-malachite green (LMG) (Sigma Aldrich, St Louis, MO, United States). Then a polyurethane resin (Crystal Clear 2006, Smooth-On, Easton, PA, USA) which was supplied in two parts (part A and part B) were mixed together by ratio of 100 to 90 to afford optically clear polyurethane resins that form the matrix of the radiochromic gel dosimeter. The solutions prepared were combined together and thoroughly mixed. Then the prepared mixture was poured into polyspectrophotometer cuvettes and cylindrical plastic containers. The filled cuvettes and containers were kept in a pressure pot (60 psi) for 5 days to minimize out gassing (3). Table 1 shows the 
percentage composition of the prepared radiochromic gel. By using mayneord formula (4) and considering the elemental compositions of radiochromic gel, the effective atomic number of the fabricated dosimeter would be 7.8 which is almost water equivalent.

Table 1. Percentage of Chemical Composition of Radiochromic Gel

\begin{tabular}{lccc}
\hline Components & Polyurethane & LMG & $\mathrm{CCl}_{\mathbf{4}}$ \\
\hline Percentage (\%) & 94 & 1 & 5 \\
\hline
\end{tabular}

LMG, leuco-malachite green.

\subsection{Absorption Spectrum}

The maximum absorptions of cuvettes were determined using UV-Vis spectrophotometer (Varian, Palo Alto, California, USA).

\subsection{Calibration}

The next step was calibration of the dosimeters against various dose levels. Varian Clinac 2100c linear accelerator was used for calibration. Various levels of absorbed doses were delivered to cuvettes. For each dose step, three cuvettes were used. All samples were kept in a dark and cold environment to prevent any accidental absorption changes and 48 hours after irradiation, again by using UVVis spectrophotometer, the maximum absorption of each cuvette was obtained. In order to ensure that the changes in light absorbance versus radiation dose are only due to the radiation doses absorbed by the dosimeter, the absorption values were obtained by subtracting the relevant values of pre-irradiation absorbance from that of the irradiated cuvettes (5). Therefore, the dose response changes and post-irradiation are solely due to the absorbed radiation doses. Finally, a calibration curve, which is the variations of optical density changes against absorbed doses, was plotted.

\subsection{Irradiating Cylindrical Radiochromic Gel Dosimeter}

After calibration and obtaining the optical density changes corresponding to each step of the absorbed dose, the irradiation of cylindrical radiochromic gel dosimeter was performed. In this section, the radiochromic gel was placed in a water phantom to prepare the electronic equilibrium condition and irradiated by three radiation fields, depicted schematically in Figure 1.

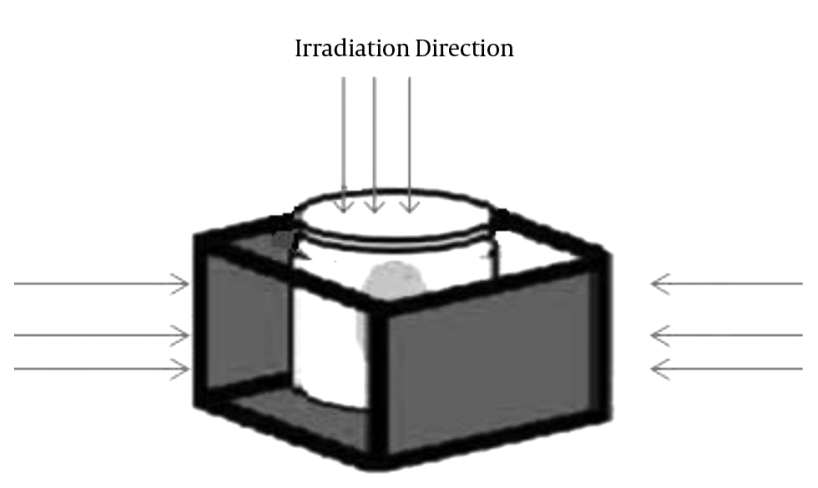

Figure 1. Schematic view of irradiation directions

\subsection{Designing Novel Optical Computed Tomography}

Initially, absorption of the radiochromic gel against various wavelengths was needed. Wavelength of the light in which maximum absorption of radiochromic gel occurred proposed the emitting light source of the novel OCT. Since maximum absorption of this gel appears at $633 \mathrm{~nm}$, which is related to red color, a 3 Watt (W) red light-emitting diode (LED) was selected as the light source. Therefore, the principle idea of this novel OCT was to measure the absorbance of the LED light that passed through the whole cylindrical radiochromic gel. The amount of absorption corresponded to the absorbed dose. In order to obtain accurate results, some other accessories were needed that are described as follows.

For such set-up, it was mandatory to make sure that all parts of the cylindrical radiochromic gel received the light of LED. Therefore, the LED was followed by a lens as optical diffuser.

Then, the cylindrical radiochromic gel was placed in a flat optical glass known as aquarium containing a matching liquid in terms of refractive index (methyl salicylate) and pinned to the step motor, providing rotation of radiochromic gel with $1.8^{\circ}$ intervals. Light emitted from the LED was collimated to a parallel beam of diameter $25 \mathrm{~cm}$ by the initial lens followed by the light source. The beam was then transmitted through the radiochromic gel, remained parallel due to existence of the matching fluid. Then a telecentric lens (TC-23-240, Opto Engineering, Italy) collected light representing attenuation line integrals onto a 12-bit charge-coupled device (CCD) array of $1040 \times 1392$ (a102f, Basler, Germany) while it rejected most of the scattered light that originated within the sample as the lens had a manufacturer specified acceptance angle less than 0.1.

In fact, telecentric optics provides a means to acquire orthographic projections; all projection lines are approxi- 
mately parallel to the optical axis, creating an ideal for parallel geometry computed tomography. To acquire a complete scan, projections are acquired at multiple angles as the radiochromic gel is rotated around $360^{\circ}$ (by the step motor).

\subsection{Dose Distribution}

In order to obtain dose distribution, multiple light beam images were taken from different directions while rotating the gel around 360 . The optical data were then fed into a tomographic reconstruction algorithm and processed by a computer. The deduction of the radiochromic gel structure from incident beam was done using a technique first obtained in 1917 by Johann Radon who was studying mathematical properties of X-ray in the tissue which is now known as Radon transform. Radon transform is an integral transform which is an extension of Beer Lambert's law whose inverse is used to reconstruct images from OCT scans. Then, conversion of optical density changes to absorbed dose is achieved through the calibration curve.

\subsection{Testing the Accuracy of the Results}

In order to verify the accuracy of the results of novel OCT, a validation test was done using a pinpoint ion chamber with an effective volume of 0.015 cc (PTW, Freiburg, Germany) and the results were then compared to those of novel OCT. In this section, the percentage depth dose and beam profile of a $3 \times 3 \mathrm{~cm}^{2}$ field size irradiating by $6 \mathrm{MV}$ photon beams were obtained by ion chamber. Then a cylindrical sample of radiochromic gel with $10 \mathrm{~cm}$ diameter was selected and irradiated to extract the percentage depth dose and beam profile.

\section{Results}

Absorption spectrum of the radiochromic gel dosimeter is shown in Figure 2. Absorption was maximum at the red region of the spectrum $(633 \mathrm{~nm})$, which is typical visible absorption of the oxidized form of LMG and it is in good agreement with previously published results $(3,6)$. Moreover, the maximum absorption wavelength is almost equal to the wavelength of $\operatorname{LED}(633 \mathrm{~nm})$, which is selected as the emitting light source of the novel OCT.

The measured absorbance changes at the wavelength of $633 \mathrm{~nm}$ versus radiation doses (the calibration curve) are displayed in Figure 3. As shown in Figure 3, a very good correlation coefficient linearity $\left(\mathrm{R}^{2}>0.99\right)$ was observed for the dose response of radiochromic gel.

By setting and arranging the previously explained accessories, the novel OCT, which is shown in Figure 4, was designed. As explained in methodology, the results of the

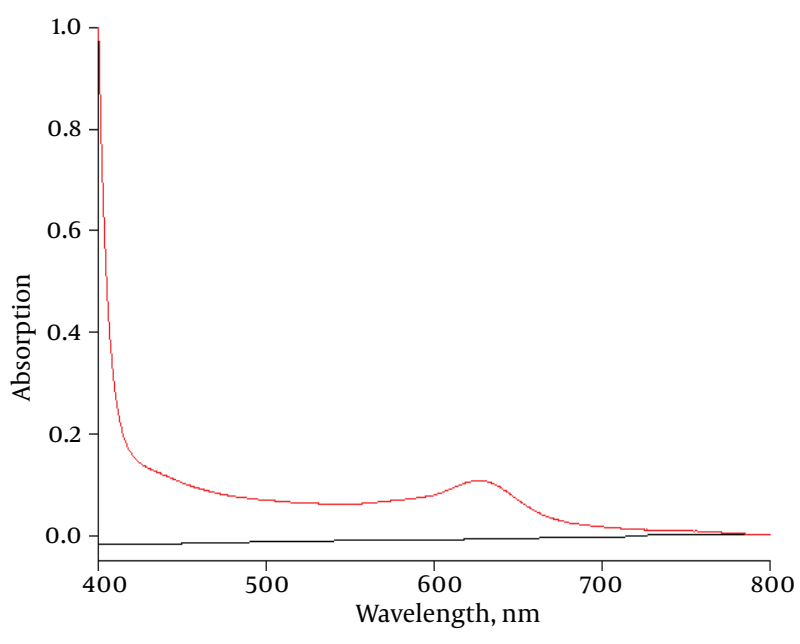

Figure 2. Absorption spectrum of radiochromic gel over visible wavelengths

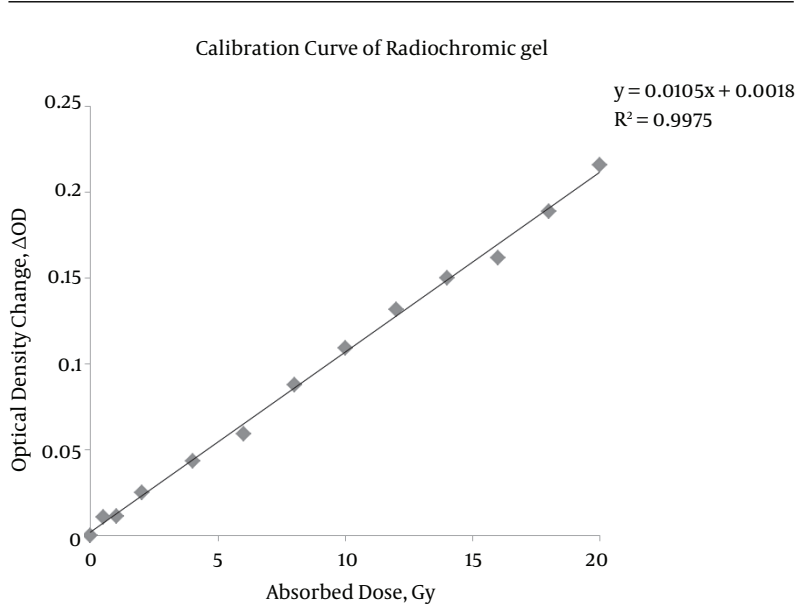

Figure 3. Calibration curve of radiochromic gel

device, which are assembled for the first time, should be verified by a precise dosimeter such as ion chamber. The best parameters for evaluating the accuracy of the results are percentage depth dose and beam profile, which show the energy deposition along the central axis and width of the radiation field, respectively. Figures 5 and 6 represent the comparison of percentage depth dose and beam profile obtained by ion chamber and the water equivalent radiochromic gel -novel OCT system. The numerical comparisons of the mentioned parameters are tabulated in Tables 2 and 3.

Figure 7 depicts the dose distribution obtained by novel-OCT. The numbers assigned to each isodose curve, represent the percentage of the absorbed doses. 
Table 2. Variation of Validation Parameter (Percentage Depth Dose) Obtained by Radiochromic Gel -Novel OCT and Ion Chamber in a $3 \times 3 \mathrm{~cm}^{2}$ Field Size

\begin{tabular}{lllcl}
\hline & Parameter & Ion Chamber & Radiochromic Gel -Novel OCT & Differences \\
\hline & Maximum difference at build up region $(\%)$ & & $3.2 \%$ \\
Percentage Depth Dose & $\mathrm{R}_{80}(\mathrm{~cm})$ & 5.5 & 3.1 & 3.8 \\
& $\mathrm{R}_{90}(\mathrm{~cm})$ & 1.6 & 1.4 \\
\hline
\end{tabular}

OCT, optical computed tomography.

Table 3. Variation of Validation Parameter (Beam Profile) Obtained by Radiochromic Gel -Novel OCT and Ion Chamber in a $3 \times 3 \mathrm{~cm}^{2}$ Field Size

\begin{tabular}{|c|c|c|c|c|}
\hline & Parameter & Ion Chamber & Radiochromic Gel-Novel OCT & Differences \\
\hline \multirow{3}{*}{ Beam Profile } & Maximum difference at flat region (\%) & & & $1.2 \%$ \\
\hline & Maximum difference at penumbra region (\%) & & & $2 \%$ \\
\hline & FWHM (Full width at half maximum) & 3 & 3.3 & \\
\hline
\end{tabular}

OCT, optical computed tomography.

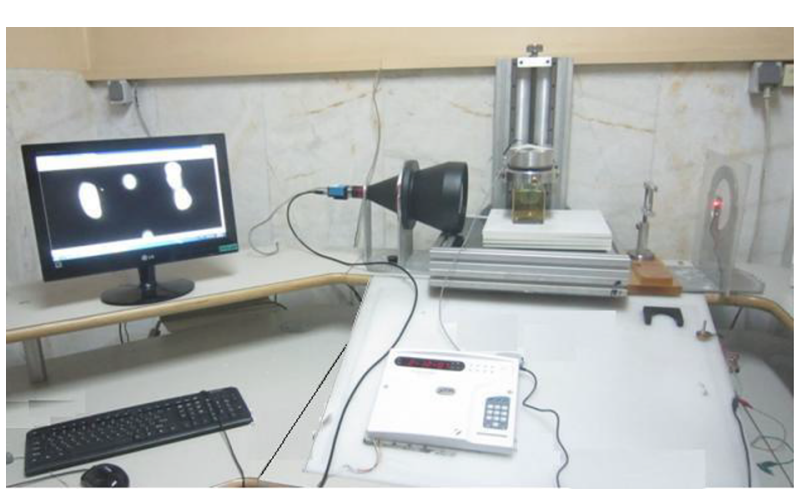

Figure 4. Novel optical computed tomography (OCT) and related accessories

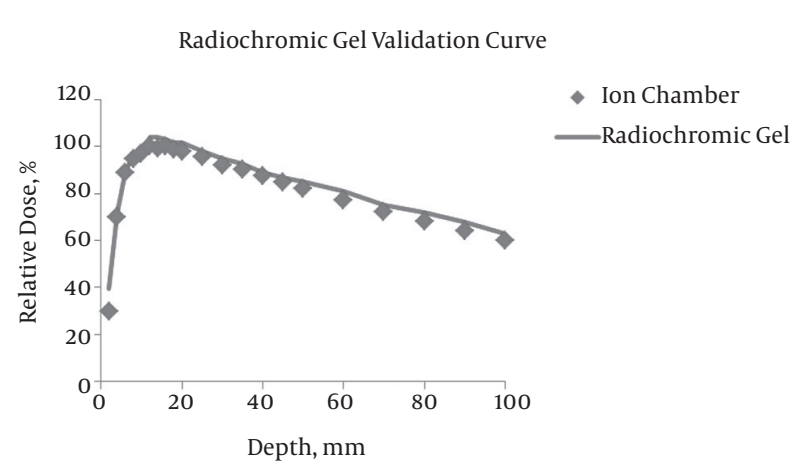

Figure 5. Comparison of percentage depth dose obtained by ion chamber and radiochromic gel -novel OCT system in a $3 \times 3 \mathrm{~cm}^{2}$ field size

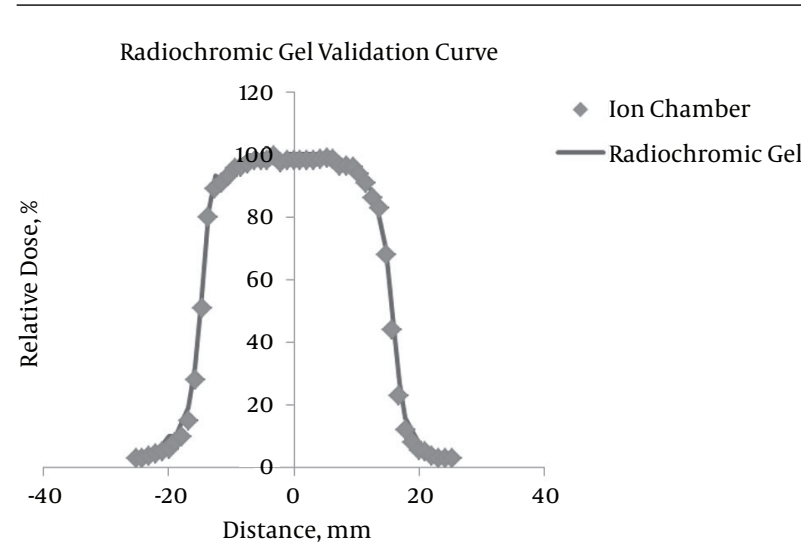

Figure 6. Comparison of beam profile obtained by ion chamber and radiochromic Gel -novel OCT system in a $3 \times 3 \mathrm{~cm}^{2}$ field size

\section{Discussion}

The maximum absorption wavelength of radiochromic gel is obtained about $633 \mathrm{~nm}$, which is completely consistent with previous studies $(3,6)$. This wavelength was the principle idea of designing a machine to extract the optical density changes occurred by means of radiation. In other words, radiochromic gel is a transparent dosimeter and ionizing radiations lead to changes in color (optical density). The calibration curve shows that the changes in optical density are linear with respect to the absorbed radiation doses (6). Therefore, finding a way to measure the optical density changes or light absorption at each point of the dosimeter would depict 


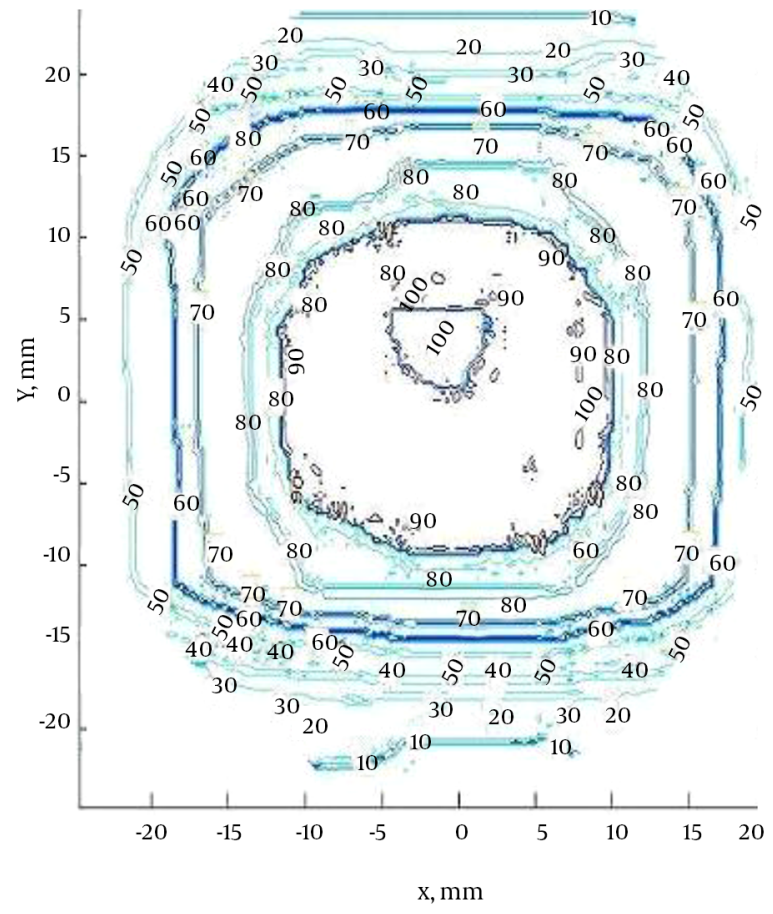

Figure 7. Dose distribution obtained by novel OCT

the dose distribution of the ionizing radiations. One of the sources that could produce the wavelength of $633 \mathrm{~nm}$ is LED. For the explained reasons, LED was chosen as the light source. Overall, the main concept of optical computed tomography is just as X-ray computed tomography (CT), but it uses visible light such as LED instead of X-ray. Therefore, by recording the images from various directions and reconstructing the projections by applying the similar methods used for image processing of CT images, the final image is created. Then by assigning the percentage of absorbed doses which are obtained by calibration curve, the dose map could be found.

Each of the mentioned accessories in designing novel OCT has a significant role in the accuracy of the results that are obtained. The existence of lens after LED converts the point light of LED to a broad and diffused band. Therefore, we can make sure that all parts of the sample (radiochromic gel) are exposed to the light emitting from LED. Matching liquid prevents the lensing and light collecting effect of radiochromic gel. Optical flat glass (aquarium) prevents refraction of the light emitting from LED. The secondary lens collects all attenuated light passing through the sample.

The accuracy test done in this study revealed that the results obtained by radiochromic gel -novel OCT could be reliable by almost $\pm 3 \%$ deviations (Tables 2 and 3 ).

Although the literature studied about the characteristics of radiochromic gel demonstrate that the dose diffusion or fading of this dosimeter is not significant, all measurements were done two days after irradiation.

To sum up, the results of this study confirmed that the Radon transform algorithm could not only be used for reconstructing $\mathrm{CT}$ images, but also applied for imaging the dose map of ionizing radiation obtained by OCT. In addition, the radiochromic gel and the novel OCT machine could be a proper set as dosimeter and read-out system for evaluating and measuring the spatial and also quantifiable dose distribution.

\section{Footnotes}

Authors' Contributions: Hassan Ali Nedaie and Alireza Zirak developed the original idea and the protocol, abstracted and analyzed the data, and wrote the manuscript. Nooshin Banaee, Alireza shirazi and sodeh sadjadi contributed to the development of the protocol, abstracted data, and prepared the manuscript.

Financial Disclosure: None declared. Funding/Support: None declared.

\section{References}

1. International Atomic Energy Agency . safety standards for protecting people and environment. Fundamental safety principles. No-SF-1. Vienna: International Atomic Energy Agency; 2006.

2. Adamovics J, Maryanski MJ, editors. A new approach to radiochromic three-dimensional dosimetry-polyurethane. J Phy: Con Se. 2004; IOP Publishing; pp. 172-5.

3. Alqathami M, Blencowe A, Qiao G, Adamovics J, Geso M. Optimizing the sensitivity and radiological properties of the PRESAGE $®$ dosimeter using metal compounds. Radiat Phys Chem. 2012;81(11):1688-95.

4. Khan FM. The Physics of Radiation Therapy. 4 ed. Philadelphia: Lippincott Williams \& Wilkins; 2010.

5. Mostaar A, Hashemi B, Zahmatkesh MH, Aghamiri SM, Mahdavi SR. A basic dosimetric study of PRESAGE: the effect of different amounts of fabricating components on the sensitivity and stability of the dosimeter. Phys Med Biol. 2010;55(3):903-12. doi: 10.1088/0031-9155/55/3/023. [PubMed: 20071770].

6. Adamovics J, Maryanski MJ. Characterisation of PRESAGE: A new 3-D radiochromic solid polymer dosemeter for ionising radiation. Radiat Prot Dosimetry. 2006;120(1-4):107-12. doi: 10.1093/rpd/nci555. [PubMed: 16782984]. 